\title{
Cardiovascular Event Rates After Myocardial Infarction or Ischaemic Stroke in Patients with Additional Risk Factors: A Retrospective Population-Based Cohort Study
}

\author{
Emil Hagström • Francesc Sorio Vilela • Maria K. Svensson • \\ Sara Hallberg · Emma Söreskog · Guillermo Villa
}

Received: April 27, 2021 / Accepted: June 29, 2021 / Published online: July 26, 2021

(c) The Author(s) 2021

\section{ABSTRACT}

Introduction: The impact of additional risk factors on major cardiovascular event (MACE) rates in patients with a history of myocardial infarction (MI) or ischaemic stroke (IS) treated with statins is not well defined.

Methods: In this retrospective populationbased cohort study, patients with a history of MI or IS treated with moderate- or high-intensity statins were identified using Swedish national register data. Patients were incident

Supplementary Information The online version contains supplementary material available at https:// doi.org/10.1007/s12325-021-01852-1.

E. Hagström ( $\varangle)$

Uppsala University Hospital, UCR-Uppsala Clinical

Research Center, 75185 Uppsala, Sweden

e-mail: emil.hagstrom@ucr.uu.se

E. Hagström · M. K. Svensson

Department of Medical Sciences, Uppsala

University, 75185 Uppsala, Sweden

F. Sorio Vilela · G. Villa

Amgen (Europe) GmbH, Suurstoffi 22,

6343 Risch-Rotkreuz, Switzerland

M. K. Svensson

Amgen AB, Gustav III:s Boulevard 54,

16974 Solna Municipality, Sweden

S. Hallberg · E. Söreskog

Quantify Research, Hantverkargatan 8,

11221 Stockholm, Sweden (index event between July 2006 and December 2014 and followed from diagnosis) or prevalent (MI or IS before July 2006 and followed thereafter). Four subgroups were defined on the basis of additional risk factors associated with increased cardiovascular risk: diabetes mellitus with target organ damage; chronic kidney disease stages 3-4; index event within 2 years after prior MI or IS; and polyvascular disease. First and total MACE rates (i.e. MI, IS, or cardiovascular death) were calculated, and first MACE 10-year risks (prevalent cohort only) were predicted.

Results: Numerically, MACE rates in subgroups were 1.5-3 times higher than in overall populations, and were highest in the 2 years after the index event. First MACE rates in the additional risk factor subgroups were $17.2-33.5$ per 100 person-years for the incident cohorts and 9.9-13.2 per 100 person-years for the prevalent cohorts. Total MACE rates per 100 person-years were 20.1-39.8 per 100 person-years and 12.4-17.6 per 100 person-years, respectively.

Conclusion: Despite previous use of moderateor high-intensity statins, patients with a history of MI or IS, and additional risk factors remain at very high cardiovascular risk.

Keywords: Cardiovascular event rates; Major cardiovascular events; Myocardial infarction; Ischaemic stroke; Lipid-lowering therapy 


\section{Key Summary Points}

Why carry out this study?

Evidence on CV event rates in patients with ASCVD comes primarily from clinical trials, so the impact of $\mathrm{CV}$ events in clinical practice may be underestimated.

We analysed Swedish national register data to estimate subsequent MACE rates over time in patients with a history of MI or IS, and additional risk factors.

\section{What was learned from this study?}

MACE rates after MI or IS were 1.5-3 times higher in patients with additional CV risk factors than in the overall MI and IS populations. Rates were highest in the 2 years after MI or IS and remained stable thereafter.

These results highlight the urgency of secondary prevention interventions early after an MI or IS to reduce the risk of subsequent MACE in these patients.

\section{INTRODUCTION}

Cardiovascular disease (CVD) is the leading cause of morbidity and mortality worldwide, placing a substantial clinical and economic burden on society [1-4]. Management of patients with CVD is based on individual risk, estimated using a combination of well-established risk factors, such as smoking, sedentary lifestyle, dyslipidaemia, hypertension, diabetes mellitus, and chronic kidney disease (CKD) [1]. In particular, elevated low-density lipoprotein cholesterol (LDL-C) is one of the most important modifiable causal factors for atherosclerotic CVD (ASCVD) [5, 6]. High LDL-C levels are directly associated with the development of ASCVD, including coronary, cerebrovascular, and peripheral artery disease (PAD). Patients at 
Death Register) were linked via unique personal identity numbers, allowing the study to use near-complete data covering the Swedish population. Data collected from the registers included diagnoses and surgical procedures from almost all hospitalisations, outpatient hospital visits and information on drugs filled at pharmacies, and all confirmed dates of death.

The study was performed in accordance with the Helsinki declaration of 1964 and its later amendments. Ethical approval for the present study was obtained from the Regional Ethical Review Board in Stockholm (dnr 2016/456-31/ 2 ). The need for individual patient consent was waived as a result of the study design.

\section{Patient Population}

The present analysis was based on a pre-existing data set that included patients aged $40-85$ years with at least one major or two minor CV risk factors, and who had previously received moderate- or high-intensity statins, with or without ezetimibe. Full details of the overall cohort have been published previously [15] and are summarised in the supplementary material online.

The timeline for patient selection and follow-up is shown in Fig. 1. For the present analysis, four cohorts of patients within the original data set were defined. Two prevalent cohorts included patients with a previously documented MI or IS, respectively, as of 1 July 2006 (index date), and who were followed from that date. Two incident cohorts included patients with a documented MI or IS, respectively, between 1 July 2006 and 31 December 2014, and who were followed from the date of the MI or IS (index date). Within each of these cohorts of patients at very high risk of MACE [1], four subgroups of patients were defined on the basis of the presence of specific additional risk factors: diabetes with target organ damage (i.e. nephropathy, retinopathy, and/or neuropathy); CKD stages 3 or 4; index event (MI or IS) within 2 years after prior MI or IS; and polyvascular disease (previous IS [MI cohort] or MI [IS cohort] and/or known PAD). Selection of these risk factors was based on current clinical guidelines [1] and the existing literature on CV risk equations $[21,22]$. Smoking status was not included in the analysis, as this information is not mandatory in the Swedish national registers and is highly under-reported. Hypertension was not included as a risk factor as it is difficult to analyse because of the challenges in creating a robust definition based only on in-hospital diagnosis codes. Similarly, pharmacotherapy use cannot be used to define hypertension, as relevant medications are also used for treating other cardiovascular diseases such as heart failure and CKD.

\section{Endpoints and Analyses}

All analyses were descriptive in nature and no formal statistical comparisons were conducted. The main endpoint was MACE, defined as a composite of MI, IS, or CV death. Patients were followed until the first event (or death or end of follow-up) for calculation of first MACE rates, and until death or end of follow-up for calculation of total MACE rates. First and total MACE rates per 100 person-years (both for the prevalent and incident cohorts) and first MACE yearly rates for years 1-6 (for the incident cohorts only) were calculated. Additionally, first MACE 10-year risks were predicted for the prevalent cohorts on the basis of exponential survival functions. A secondary endpoint, a composite of MACE, coronary revascularisation,

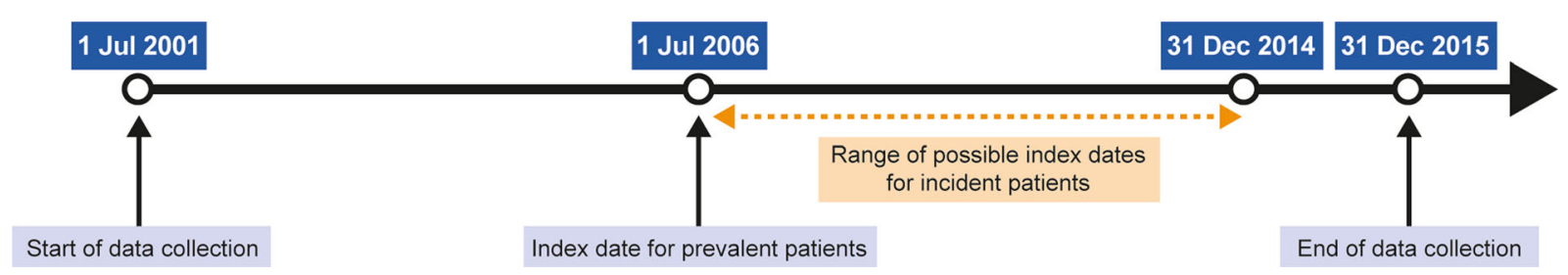

Fig. 1 Timeline of data collection and follow-up 
or unstable angina, was also considered. All statistical analyses were performed using Stata 16 (StataCorp LP, College Station, TX, USA).

\section{RESULTS}

\section{Study Population}

In total, 45,895 incident patients and 37,480 prevalent patients with a history of $\mathrm{MI}$, and 36,134 incident patients and 19,024 prevalent patients with history of IS were included in the analyses (Fig. 2). Baseline characteristics of the incident and prevalent MI and IS cohorts are shown in Tables 1 and 2. As expected, patients in the incident cohorts had a higher prevalence of prior $\mathrm{CV}$ events and additional risk factors compared with the prevalent cohorts. The mean time from qualifying MI to index date in the prevalent MI cohort was 2.1 years, and the mean time from qualifying IS to index date in the prevalent IS cohort was 1.9 years.

\section{MACE Rates}

First MACE rates per 100 person-years in patients with history of MI were 11.9 in the incident cohort and 6.2 in the prevalent cohort (Table 3). Rates in the subgroups with additional risk factors were numerically $2-3$ times higher than in the overall population for the incident MI cohort (21.3-33.5 per 100 personyears) and 1.5-2 times higher for the prevalent MI cohort (10.7-13.2 per 100 person-years). In the prevalent MI cohort, the predicted first MACE 10-year risk in the subgroups with additional risk factors (66-73\%) was also substantially higher than in the overall prevalent MI population (46\%) (Table 3).

In patients with prior IS, first MACE rates per 100 person-years were 12.3 and 6.9 in the incident and prevalent cohorts, respectively

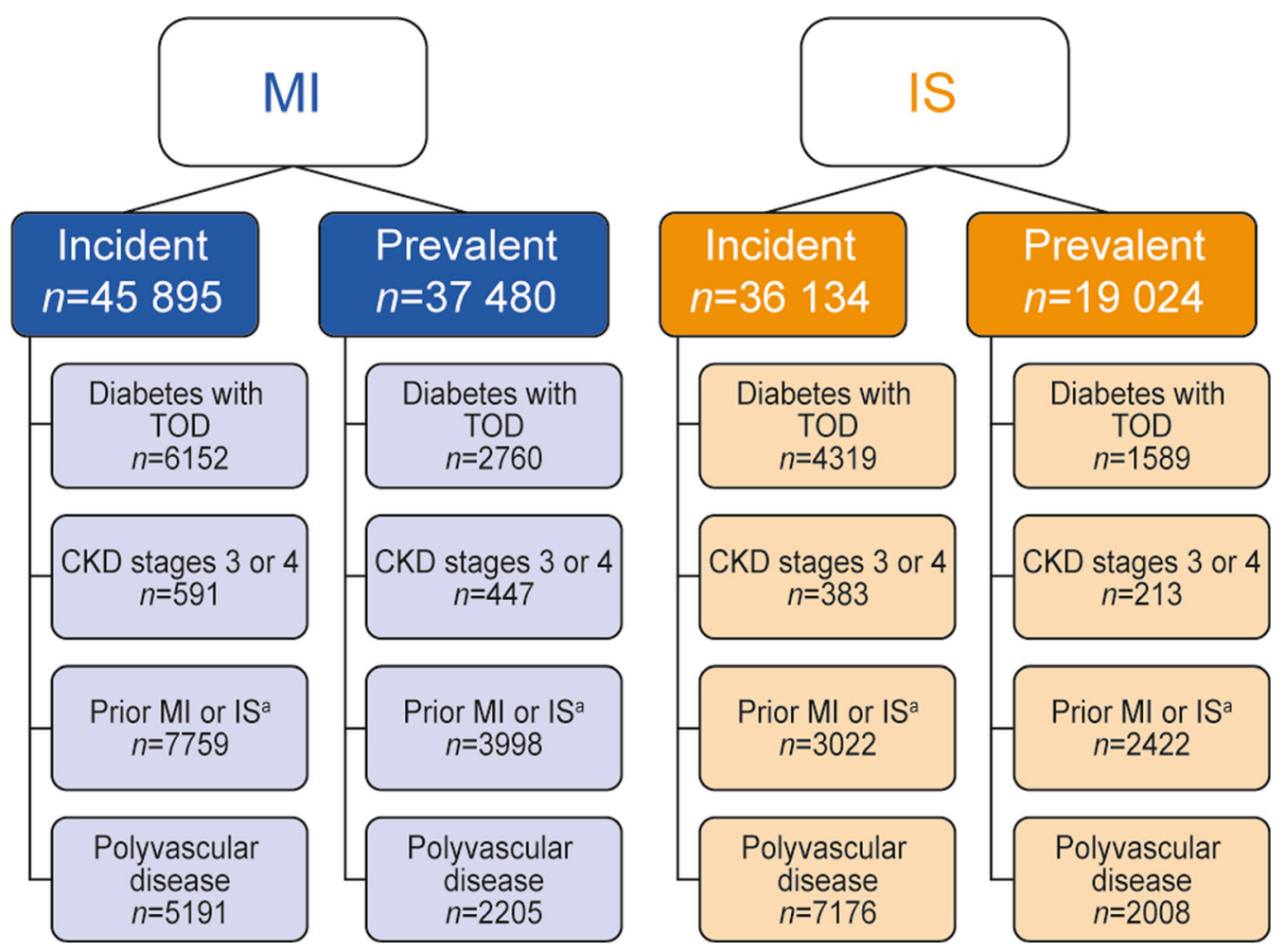

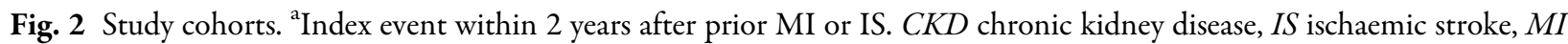
myocardial infarction, $T O D$ target organ damage 
Table 1 Baseline characteristics of patients with a history of MI, overall and subgroups

\begin{tabular}{|c|c|c|c|c|c|c|c|c|c|}
\hline \multirow[t]{2}{*}{ Incident MI cohort } & \multirow{2}{*}{\multicolumn{2}{|c|}{$\begin{array}{l}\text { Overall } \\
(n=45,895)\end{array}$}} & \multicolumn{7}{|c|}{ Subgroups } \\
\hline & & & \multicolumn{2}{|c|}{$\begin{array}{l}\text { Diabetes with } \\
\text { TOD } \\
(n=6152)\end{array}$} & \multicolumn{2}{|c|}{$\begin{array}{l}\text { CKD stages } 3 \\
\text { or } 4(n=591)\end{array}$} & \multicolumn{2}{|c|}{$\begin{array}{l}\text { MI within } 2 \text { years after } \\
\text { prior MI or IS } \\
(n=7759)\end{array}$} & $\begin{array}{l}\text { Polyvascular } \\
\text { disease } \\
(n=5191)\end{array}$ \\
\hline $\begin{array}{l}\text { Follow-up (years), } \\
\text { mean (SD) }\end{array}$ & \multicolumn{2}{|l|}{$3.9(2.7)$} & \multicolumn{2}{|l|}{$3.1(2.6)$} & \multicolumn{2}{|l|}{$1.7(1.5)$} & \multicolumn{2}{|l|}{$3.9(2.7)$} & $3.0(2.5)$ \\
\hline $\begin{array}{l}\text { Age (years), mean } \\
(\mathrm{SD})\end{array}$ & \multicolumn{2}{|c|}{$71.0(9.6)$} & \multicolumn{2}{|l|}{$70.7(9.4)$} & \multicolumn{2}{|l|}{$72.9(8.3)$} & \multicolumn{2}{|l|}{$73.4(9.0)$} & $74.2(8.0)$ \\
\hline Male sex, $n(\%)$ & \multicolumn{2}{|c|}{$30,704(67)$} & \multicolumn{2}{|c|}{$3833(62)$} & \multicolumn{2}{|l|}{$426(72)$} & \multicolumn{2}{|l|}{$4840(62)$} & $3366(65)$ \\
\hline \multicolumn{10}{|l|}{ CV history, $n$ (\%) } \\
\hline CABG/PCI & \multicolumn{2}{|c|}{$9819(21)$} & \multicolumn{2}{|c|}{$2313(38)$} & \multicolumn{2}{|l|}{$245(41)$} & \multicolumn{2}{|l|}{$3175(41)$} & $1808(35)$ \\
\hline IS & \multicolumn{2}{|c|}{$3981(9)$} & \multicolumn{2}{|l|}{$756(12)$} & \multicolumn{2}{|l|}{$68(12)$} & \multicolumn{2}{|l|}{$2026(26)$} & $4545(88)$ \\
\hline PAD & $2815(6)$ & & $156(3)$ & & $13(2)$ & & $127(2)$ & & $746(14)$ \\
\hline $\begin{array}{l}\text { Charlson } \\
\text { comorbidity } \\
\text { index, }{ }^{a} \text { mean }(\mathrm{SD})\end{array}$ & $3.2(2.2)$ & & $5.5(1.8)$ & & $6.6(2.3)$ & & $3.5(2.1)$ & & $4.2(2.1)$ \\
\hline Additional risk factor & $\mathrm{s}, n(\%)$ & & & & & & & & \\
\hline Hypertension & $31,172($ & & $3300(54$ & & $500(85)$ & & $3958(51)$ & & $3170(61)$ \\
\hline Diabetes & 19,699 & & $6152(10$ & & $351(59)$ & & $3621(47)$ & & $2219(43)$ \\
\hline CKD & $3261(7)$ & & $864(14)$ & & $591(100)$ & & $514(7)$ & & $327(6)$ \\
\hline Lipid-lowering therap & $y$ at index & date, & $n(\%)$ & & & & & & \\
\hline $\begin{array}{l}\text { Moderate- or high- } \\
\text { intensity statin }\end{array}$ & $33,664($ & & $4287(70$ & & $342(58)$ & & $4512(58)$ & & $2616(50)$ \\
\hline $\begin{array}{l}\text { Low-intensity } \\
\text { statin }\end{array}$ & $2649(6)$ & & $835(14)$ & & $84(14)$ & & $989(13)$ & & $605(12)$ \\
\hline Ezetimibe & $931(2)$ & & $148(2)$ & & $26(4)$ & & $185(2)$ & & $98(2)$ \\
\hline Prevalent MI cohort & & Over & & Subgr & & & & & \\
\hline & & & $37,480)$ & $\begin{array}{l}\text { Diabe } \\
\text { TOD }\end{array}$ & $\begin{array}{l}\text { s with } \\
n=2760)\end{array}$ & $\begin{array}{l}\text { CKD } \\
\text { or } 4\end{array}$ & $\begin{array}{l}\text { stages } 3 \\
(n=447)\end{array}$ & $\begin{array}{l}\text { MI within } 2 \text { years } \\
\text { after prior MI } \\
\text { or IS }(n=3998)\end{array}$ & $\begin{array}{l}\text { Polyvascular } \\
\text { disease } \\
(n=2205)\end{array}$ \\
\hline Follow-up (years), me & an $(S D)$ & $7.3(3$ & & $5.9(3$. & & $3.6(1$ & 1.7) & $6.2(3.4)$ & $5.9(3.3)$ \\
\hline Age (years), mean (SI & & 72.3 & $(8.5)$ & 69.3( & & 73.4 & (8.8) & $72.3(9.8)$ & $73.6(8.5)$ \\
\hline Male sex, $n(\%)$ & & 24,60 & $8(66)$ & 1753 & & 317 & (71) & $2620(66)$ & $1423(65)$ \\
\hline CV history, $n$ (\%) & & & & & & & & & \\
\hline CABG/PCI & & 3977 & (11) & $947(3$ & & 234( & (52) & $1778(44)$ & $683(31)$ \\
\hline
\end{tabular}


Table 1 continued

\begin{tabular}{|c|c|c|c|c|c|}
\hline \multirow[t]{2}{*}{ Prevalent MI cohort } & \multirow{2}{*}{$\begin{array}{l}\text { Overall } \\
(n=37,480)\end{array}$} & \multicolumn{4}{|l|}{ Subgroups } \\
\hline & & $\begin{array}{l}\text { Diabetes with } \\
\text { TOD }(n=2760)\end{array}$ & $\begin{array}{l}\text { CKD stages } 3 \\
\text { or } 4(n=447)\end{array}$ & $\begin{array}{l}\text { MI within } 2 \text { years } \\
\text { after prior MI } \\
\text { or IS }(n=3998)\end{array}$ & $\begin{array}{l}\text { Polyvascular } \\
\text { disease } \\
(n=2205)\end{array}$ \\
\hline IS & $2852(8)$ & $215(8)$ & $27(6)$ & $721(18)$ & $1913(87)$ \\
\hline $\mathrm{PAD}$ & $384(1)$ & $66(2)$ & $6(1)$ & $54(1)$ & $311(14)$ \\
\hline $\begin{array}{l}\text { Charlson comorbidity } \\
\text { index, }{ }^{a} \text { mean }(\mathrm{SD})\end{array}$ & $2.6(1.9)$ & $5.1(1.6)$ & $5.7(1.9)$ & $2.9(1.9)$ & $3.6(1.8)$ \\
\hline \multicolumn{6}{|c|}{ Additional risk factors, $n(\%)$} \\
\hline Hypertension & $16,755(45)$ & $946(34)$ & $315(70)$ & $1438(36)$ & $934(42)$ \\
\hline Diabetes & $13,633(36)$ & $2760(100)$ & $220(49)$ & $1234(31)$ & $757(34)$ \\
\hline $\mathrm{CKD}$ & $434(1)$ & $263(10)$ & $447(100)$ & $168(4)$ & $79(4)$ \\
\hline \multicolumn{6}{|l|}{$\begin{array}{l}\text { Lipid-lowering therapy } \\
\text { at index date, } n(\%)\end{array}$} \\
\hline $\begin{array}{l}\text { Moderate- or } \\
\text { high-intensity statin }\end{array}$ & $36,290(97)$ & $2450(89)$ & $321(72)$ & $2123(53)$ & $1939(88)$ \\
\hline Low-intensity statin & $815(2)$ & $66(2)$ & $40(9)$ & $104(3)$ & $56(3)$ \\
\hline Ezetimibe & $563(2)$ & $56(2)$ & $16(4)$ & $83(2)$ & $30(1)$ \\
\hline
\end{tabular}

CABG/PCI coronary artery bypass graft/percutaneous coronary intervention, $C K D$ chronic kidney disease, $C V$ cardiovascular, $I S$ ischaemic stroke, $M I$ myocardial infarction, $P A D$ peripheral artery disease, $S D$ standard deviation, $T I A$ transient ischemic attack, $T O D$ target organ damage

a The Charlson comorbidity index is a weighted index that takes into account the number and seriousness of comorbid diseases [29]

(Table 4). Rates in the subgroups with specific additional risk factors were numerically more than 1.5 times higher than in the respective overall population for the incident IS cohorts (17.2-18.8 per 100 person-years) and $1.5-2$ times higher in the prevalent IS cohorts (9.9-12.3 per 100 person-years). In the prevalent IS cohort, the predicted first MACE 10-year risk in the subgroups with specific additional risk factors (63-71\%) was also substantially higher than in the overall prevalent IS population (50\%) (Table 4). Results were similar, but with higher rates, for the secondary endpoint of the composite of MACE, coronary revascularisation, or unstable angina (Table S1 in the supplementary material).
Total MACE rates per 100 person-years were 15.4 and 8.4 in the overall incident and prevalent MI cohorts, respectively (27.2-39.8 per 100 person-years and $15.4-17.6$ per 100 personyears, respectively, in the subgroups) (Table 3). Total MACE rates were 14.4 and 7.5 per 100 person-years in the overall incident and prevalent IS cohorts (subgroups: 20.1-23.9 and 12.4-17.0 per 100 person-years, respectively) (Table 4).

\section{MACE Rates Over Time}

Across all subgroups within the incident MI and IS cohorts, MACE rates were highest in the first year after the index event, declining during year 2 and remaining stable thereafter (Fig. 3). 
Table 2 Baseline characteristics of patients with a history of IS, overall and subgroups

\begin{tabular}{|c|c|c|c|c|c|c|c|}
\hline \multirow[t]{2}{*}{ Incident IS cohort } & \multirow{2}{*}{$\begin{array}{l}\text { Overall } \\
(n=36,134)\end{array}$} & \multicolumn{6}{|l|}{ Subgroups } \\
\hline & & $\begin{array}{l}\text { Diabetes with } \\
\text { TOD } \\
(n=4319)\end{array}$ & \multicolumn{2}{|c|}{$\begin{array}{l}\text { CKD stages } 3 \\
\text { or } 4(n=383)\end{array}$} & \multicolumn{2}{|c|}{$\begin{array}{l}\text { MI within } 2 \text { years after } \\
\text { prior MI or IS } \\
(n=3022)\end{array}$} & $\begin{array}{l}\text { Polyvascular } \\
\text { disease } \\
(n=7176)\end{array}$ \\
\hline $\begin{array}{l}\text { Follow-up (years), } \\
\text { mean (SD) }\end{array}$ & $3.7(2.6)$ & $3.2(2.5)$ & \multicolumn{2}{|l|}{$1.5(2.0)$} & \multicolumn{2}{|l|}{$3.8(2.8)$} & $3.2(2.5)$ \\
\hline $\begin{array}{l}\text { Age (years), mean } \\
\text { (SD) }\end{array}$ & $72.9(8.7)$ & $71.7(8.9)$ & \multicolumn{2}{|c|}{$73.7(8.4)$} & \multicolumn{2}{|l|}{$73.8(8.7)$} & $74.5(8.1)$ \\
\hline Male sex, $n(\%)$ & $27,719(60)$ & $2743(64)$ & \multicolumn{2}{|l|}{$269(70)$} & $1839(61)$ & & $4741(66)$ \\
\hline \multicolumn{8}{|l|}{ CV history, $n$ (\%) } \\
\hline TIA & $4048(11)$ & $466(11)$ & \multicolumn{2}{|l|}{$47(12)$} & $400(13)$ & & $754(11)$ \\
\hline MI & $5321(15)$ & $844(20)$ & \multicolumn{2}{|l|}{$52(14)$} & $2145(71)$ & & $6822(95)$ \\
\hline $\mathrm{PAD}$ & $1624(5)$ & $85(2)$ & \multicolumn{2}{|l|}{$7(2)$} & $31(1)$ & & $306(4)$ \\
\hline $\begin{array}{l}\text { Charlson } \\
\text { comorbidity } \\
\text { index, }{ }^{\mathrm{a}} \text { mean }(\mathrm{SD})\end{array}$ & $3.0(2.1)$ & $5.4(1.9)$ & \multicolumn{2}{|l|}{$6.4(2.3)$} & $3.5(2.1)$ & & $4.1(2.1)$ \\
\hline \multicolumn{8}{|c|}{ Additional risk factors, $n(\%)$} \\
\hline Hypertension & $26,535(73)$ & $2433(56)$ & \multicolumn{2}{|l|}{$320(84)$} & $1731(57)$ & & $4121(57)$ \\
\hline Diabetes & $14,140(39)$ & $4319(100)$ & \multicolumn{2}{|l|}{$203(53)$} & $1027(34)$ & & $2584(36)$ \\
\hline CKD & $1772(5)$ & $434(10)$ & \multicolumn{2}{|c|}{$383(100)$} & $119(4)$ & & $341(5)$ \\
\hline \multicolumn{8}{|c|}{ Lipid-lowering therapy at index date, $n$ (\%) } \\
\hline $\begin{array}{l}\text { Moderate- or high- } \\
\text { intensity statin }\end{array}$ & $31,386(87)$ & $3795(88)$ & \multicolumn{2}{|l|}{$336(88)$} & $2559(85)$ & & $6457(90)$ \\
\hline $\begin{array}{l}\text { Low-intensity } \\
\text { statin }\end{array}$ & $2113(6)$ & $647(15)$ & $61(16)$ & & $271(9)$ & & $670(9)$ \\
\hline Ezetimibe & $448(1)$ & $61(1)$ & $7(2)$ & & $39(1)$ & & $124(2)$ \\
\hline Prevalent IS cohort & & Overall & Subgroups & & & & \\
\hline & & 19,024) & $\begin{array}{l}\text { Diabetes } \\
\text { with TOD } \\
(n=1589)\end{array}$ & $\begin{array}{l}\text { CKD } s \\
3 \text { or } 4\end{array}$ & $\begin{array}{l}\text { stages } \\
(n=213)\end{array}$ & $\begin{array}{l}\text { MI within } 2 \text { years } \\
\text { after prior MI } \\
\text { or IS }(n=2422)\end{array}$ & $\begin{array}{ll}\text { rs } & \begin{array}{l}\text { Polyvascular } \\
\text { disease }\end{array} \\
(n=2008)\end{array}$ \\
\hline Follow-up (years), me & an $(S D)$ & $7.0(3.1)$ & $6.0(3.3)$ & $3.5(1.8$ & & $6.6(3.2)$ & $5.9(3.3)$ \\
\hline Age (years), mean (SI & & $73.0(7.7)$ & $69.7(8.8)$ & $73.3(8$. & 3.3) & $71.9(9.0)$ & $73.8(8.3)$ \\
\hline Male sex, $n(\%)$ & & $11201(59)$ & $1008(63)$ & $170(80$ & & $1540(64)$ & $1297(65)$ \\
\hline CV history, $n$ (\%) & & & & & & & \\
\hline TIA & & $1894(10)$ & $150(9)$ & $24(11)$ & & $316(13)$ & $193(10)$ \\
\hline
\end{tabular}


Table 2 continued

\begin{tabular}{|c|c|c|c|c|c|}
\hline \multirow[t]{2}{*}{ Prevalent IS cohort } & \multirow{2}{*}{$\begin{array}{l}\text { Overall } \\
(n=19,024)\end{array}$} & \multicolumn{4}{|l|}{ Subgroups } \\
\hline & & $\begin{array}{l}\text { Diabetes } \\
\text { with TOD } \\
(n=1589)\end{array}$ & $\begin{array}{l}\text { CKD stages } \\
3 \text { or } 4(n=213)\end{array}$ & $\begin{array}{l}\text { MI within } 2 \text { years } \\
\text { after prior MI } \\
\text { or IS }(n=2422)\end{array}$ & $\begin{array}{l}\text { Polyvascular } \\
\text { disease } \\
(n=2008)\end{array}$ \\
\hline MI & $2852(15)$ & $228(14)$ & $28(13)$ & $770(32)$ & $1913(95)$ \\
\hline PAD & $131(1)$ & $17(1)$ & $-{ }^{b}$ & $22(1)$ & $114(6)$ \\
\hline $\begin{array}{l}\text { Charlson comorbidity } \\
\text { index, }{ }^{a} \text { mean }(\mathrm{SD})\end{array}$ & $2.5(1.8)$ & $4.7(1.5)$ & $5.4(2.2)$ & $2.6(1.7)$ & $3.6(1.7)$ \\
\hline \multicolumn{6}{|l|}{ Additional risk factors, $n(\%)$} \\
\hline Hypertension & $8120(43)$ & $632(40)$ & $165(78)$ & $1106(46)$ & $861(43)$ \\
\hline Diabetes & $7026(37)$ & $1589(100)$ & $98(46)$ & $723(30)$ & $674(34)$ \\
\hline $\mathrm{CKD}$ & $436(2)$ & $121(8)$ & $213(100)$ & $44(2)$ & $68(3)$ \\
\hline \multicolumn{6}{|l|}{$\begin{array}{l}\text { Lipid-lowering therapy } \\
\text { at index date, } n(\%)\end{array}$} \\
\hline Moderate- or high-intensity statin & $13,880(73)$ & $1383(87)$ & $86(40)$ & $2140(88)$ & $1757(88)$ \\
\hline Low-intensity statin & $981(5)$ & $44(3)$ & $18(8)$ & $69(3)$ & $54(3)$ \\
\hline Ezetimibe & $142(1)$ & $23(1)$ & $-{ }^{\mathrm{b}}$ & $31(1)$ & $31(2)$ \\
\hline
\end{tabular}

$C A B G / P C I$ coronary artery bypass graft/percutaneous coronary intervention, $C K D$ chronic kidney disease, $C V$ cardiovascular, $I S$ ischaemic stroke, $M I$ myocardial infarction, $P A D$ peripheral artery disease, $S D$ standard deviation, $T I A$ transient ischemic attack, TOD target organ damage

a The Charlson comorbidity index is a weighted index that takes into account the number and seriousness of comorbid diseases [29]

b $n<5$, data not shown

\section{DISCUSSION}

This large study with nationwide coverage of Swedish register data shows that patients with a history of MI or IS, and additional risk factors have a very high risk of suffering subsequent MACE despite previous use of standard-of-care lipid-lowering therapy (i.e. moderate- or highintensity statins, with or without ezetimibe). This is particularly true for patients with specific additional risk factors, such as diabetes, CKD, subsequent events within 2 years, and polyvascular disease.

Patients in the prevalent cohorts may have had their qualifying MI or IS event up to 5 years before the index date, while patients in the incident cohorts were followed from the time of the qualifying event. This led to differences in MACE rates between the two cohorts, as the risk of a second event is highest in the first years after the initial event and decreases thereafter. The risk of a subsequent $\mathrm{CV}$ event has been reported to be higher in the first year post-MI and remain high in the following years [1], and this study aimed to describe the change in CV risk over time by producing MACE yearly rates for the incident cohorts.

In patients with an incident MI or IS, first MACE rates were highest in the first 2 years after the index event, decreasing during year 2 and remaining stable in year 3 and beyond. At 1 year after the index event, the rates were more than three times that seen after year 3 across all subgroups. Moreover, in the first year after MI or IS, the MACE yearly rates in the subgroups of 
Table 3 First and total MACE rates of patients with a history of MI, overall and subgroups

\begin{tabular}{|c|c|c|c|c|c|c|c|c|}
\hline & \multicolumn{5}{|c|}{ First MACE } & \multicolumn{3}{|c|}{ Total MACE } \\
\hline & $n$ & $\begin{array}{l}\text { Events } \\
(n)\end{array}$ & $\begin{array}{l}\text { Follow-up } \\
\text { (person- } \\
\text { years) }\end{array}$ & $\begin{array}{l}\text { MACE rate per } \\
100 \text { person- } \\
\text { years }\end{array}$ & $\begin{array}{l}\text { 10-year } \\
\text { risk } \\
(\%)\end{array}$ & $\begin{array}{l}\text { Events } \\
(n)\end{array}$ & $\begin{array}{l}\text { Follow-up } \\
\text { (person- } \\
\text { years) }\end{array}$ & $\begin{array}{l}\text { MACE rate per } \\
100 \text { person- } \\
\text { years }\end{array}$ \\
\hline \multicolumn{9}{|c|}{ Incident MI cohort } \\
\hline Overall & 45,895 & 18,021 & 151,317 & 11.9 & - & 27,255 & 177,057 & 15.4 \\
\hline Subgroups & & & & & - & & & \\
\hline $\begin{array}{l}\text { Diabetes } \\
\text { with TOD }\end{array}$ & 6152 & 3179 & 14,910 & 21.3 & & 5209 & 19,172 & 27.2 \\
\hline $\begin{array}{l}\text { CKD } \\
\text { stages } 3 \text { or } 4\end{array}$ & 591 & 281 & 839 & 33.5 & & 397 & 998 & 39.8 \\
\hline $\begin{array}{l}\text { Prior MI or } \\
\text { IS }^{a}\end{array}$ & 7759 & 2065 & 9467 & 21.8 & & 3545 & 12,711 & 27.9 \\
\hline $\begin{array}{l}\text { Polyvascular } \\
\text { disease }\end{array}$ & 5191 & 842 & 3301 & 25.5 & & 4291 & 15,757 & 27.2 \\
\hline \multicolumn{9}{|c|}{ Prevalent MI cohort } \\
\hline Overall & 37,480 & 15,100 & 245,177 & 6.2 & 46 & 23,102 & 275,117 & 8.4 \\
\hline \multicolumn{9}{|l|}{ Subgroups } \\
\hline $\begin{array}{l}\text { Diabetes } \\
\text { with TOD }\end{array}$ & 2760 & 1465 & 13,654 & 10.7 & 66 & 2848 & 16,241 & 17.5 \\
\hline $\begin{array}{l}\text { CKD } \\
\text { stages } 3 \text { or } 4\end{array}$ & 447 & 189 & 1431 & 13.2 & 73 & 281 & 1597 & 17.6 \\
\hline $\begin{array}{l}\text { Prior MI or } \\
\text { IS }^{\mathrm{a}}\end{array}$ & 3998 & 2218 & 20,511 & 10.8 & 66 & 3806 & 24,762 & 15.4 \\
\hline $\begin{array}{l}\text { Polyvascular } \\
\text { disease }\end{array}$ & 2205 & 1279 & 10,947 & 11.7 & 69 & 2037 & 13,017 & 15.6 \\
\hline
\end{tabular}

$C K D$ chronic kidney disease, $I S$ ischaemic stroke, $M A C E$ major cardiovascular events, $M I$ myocardial infarction, $T O D$ target organ damage

${ }^{a}$ Index event within 2 years after prior MI or IS

patients with specific additional risk factors were almost double those of the overall incident MI or IS cohorts.

As expected, first MACE rates in the present study based on real-world clinical practice data were considerably higher than those seen in recent clinical trials. Notably, this data set was designed to have almost identical inclusion criteria to the FOURIER study [23]. To allow a comparison of real-world and clinical trial data, we conducted an ad hoc analysis of data from the FOURIER study, showing that first MACE rates in the placebo arm in patients with a history of MI were 3.4 per 100 person-years overall, and 3.8-6.3 per 100 person-years in the subgroups with additional risk factors (Table S2 in the supplementary material) (Amgen, data on file), compared with 6.2 per 100 person-years 
Table 4 First and total MACE rates of patients with a history of IS, overall and subgroups

\begin{tabular}{|c|c|c|c|c|c|c|c|c|}
\hline & \multirow[t]{2}{*}{$n$} & \multicolumn{4}{|c|}{ First MACE } & \multicolumn{3}{|c|}{ Total MACE } \\
\hline & & $\begin{array}{l}\text { Events } \\
(n)\end{array}$ & $\begin{array}{l}\text { Follow-up } \\
\text { (person- } \\
\text { years) }\end{array}$ & $\begin{array}{l}\text { MACE rate per } \\
100 \text { person- } \\
\text { years }\end{array}$ & $\begin{array}{l}\text { 10-year } \\
\text { risk } \\
(\%)\end{array}$ & $\begin{array}{l}\text { Events } \\
(n)\end{array}$ & $\begin{array}{l}\text { Follow-up } \\
\text { (person- } \\
\text { years) }\end{array}$ & $\begin{array}{l}\text { MACE rate per } \\
100 \text { person- } \\
\text { years }\end{array}$ \\
\hline \multicolumn{9}{|c|}{ Incident IS cohort } \\
\hline Overall & 36,134 & 14,039 & 113,982 & 12.3 & - & 19,058 & 132,189 & 14.4 \\
\hline Subgroups & & & & & & & & \\
\hline $\begin{array}{l}\text { Diabetes } \\
\text { with TOD }\end{array}$ & 4319 & 1969 & 11,448 & 17.2 & - & 2778 & 13,850 & 20.1 \\
\hline $\begin{array}{l}\text { CKD } \\
\text { stages } 3 \text { or } 4\end{array}$ & 383 & 259 & 1423 & 18.2 & - & 392 & 1798 & 21.8 \\
\hline $\begin{array}{l}\text { Prior MI or } \\
\mathrm{IS}^{\mathrm{a}}\end{array}$ & 3022 & 1672 & 8888 & 18.8 & - & 2736 & 11,455 & 23.9 \\
\hline $\begin{array}{l}\text { Polyvascular } \\
\text { disease }\end{array}$ & 7176 & 3440 & 19,046 & 18.1 & - & 4816 & 22,775 & 21.1 \\
\hline \multicolumn{9}{|c|}{ Prevalent IS cohort } \\
\hline Overall & 19,024 & 8124 & 118,272 & 6.9 & 50 & 9990 & 132,849 & 7.5 \\
\hline Subgroups & & & & & & & & \\
\hline $\begin{array}{c}\text { Diabetes } \\
\text { with TOD }\end{array}$ & 1589 & 796 & 8036 & 9.9 & 63 & 1282 & 9512 & 13.5 \\
\hline $\begin{array}{l}\text { CKD } \\
\text { stages } 3 \text { or } 4\end{array}$ & 213 & 81 & 661 & 12.3 & 71 & 118 & 745 & 15.8 \\
\hline $\begin{array}{l}\text { Prior MI or } \\
\text { IS }^{\mathrm{a}}\end{array}$ & 2422 & 1227 & 12,386 & 9.9 & 63 & 1865 & 14,990 & 12.4 \\
\hline $\begin{array}{l}\text { Polyvascular } \\
\text { disease }\end{array}$ & 2008 & 1175 & 9850 & 11.9 & 70 & 2000 & 11,739 & 17.0 \\
\hline
\end{tabular}

$C K D$ chronic kidney disease, IS ischaemic stroke, MACE major cardiovascular events, $M I$ myocardial infarction, TOD target organ damage

a Index event within 2 years after prior MI or IS

overall and 10.7-13.2 per 100 person-years in the subgroups for prevalent patients in the present study. In patients with a recent MI (within less than 1 year) in the placebo arm of FOURIER, the first MACE rate was 3.8 per 100 person-years, compared with 11.9 per 100 person-years in the incident MI cohort in the present study. Similarly, in the ODYSSEY study, 1126 MACE events (but also including non-CV mortality) were reported in 9462 patients with a recent acute coronary syndrome followed for 2.8 years, meaning an event rate of 4.3 per 100 person-years for the placebo arm [14]. First MACE rates in patients with a history of IS in the placebo arm of FOURIER were 4.0 per 100 person-years overall and 4.7-6.7 per 100 personyears in the subgroups, compared with 6.9 and 9.9-12.3 per 100 person-years, respectively, in prevalent patients in the present analysis (Table S2 in the supplementary material) 

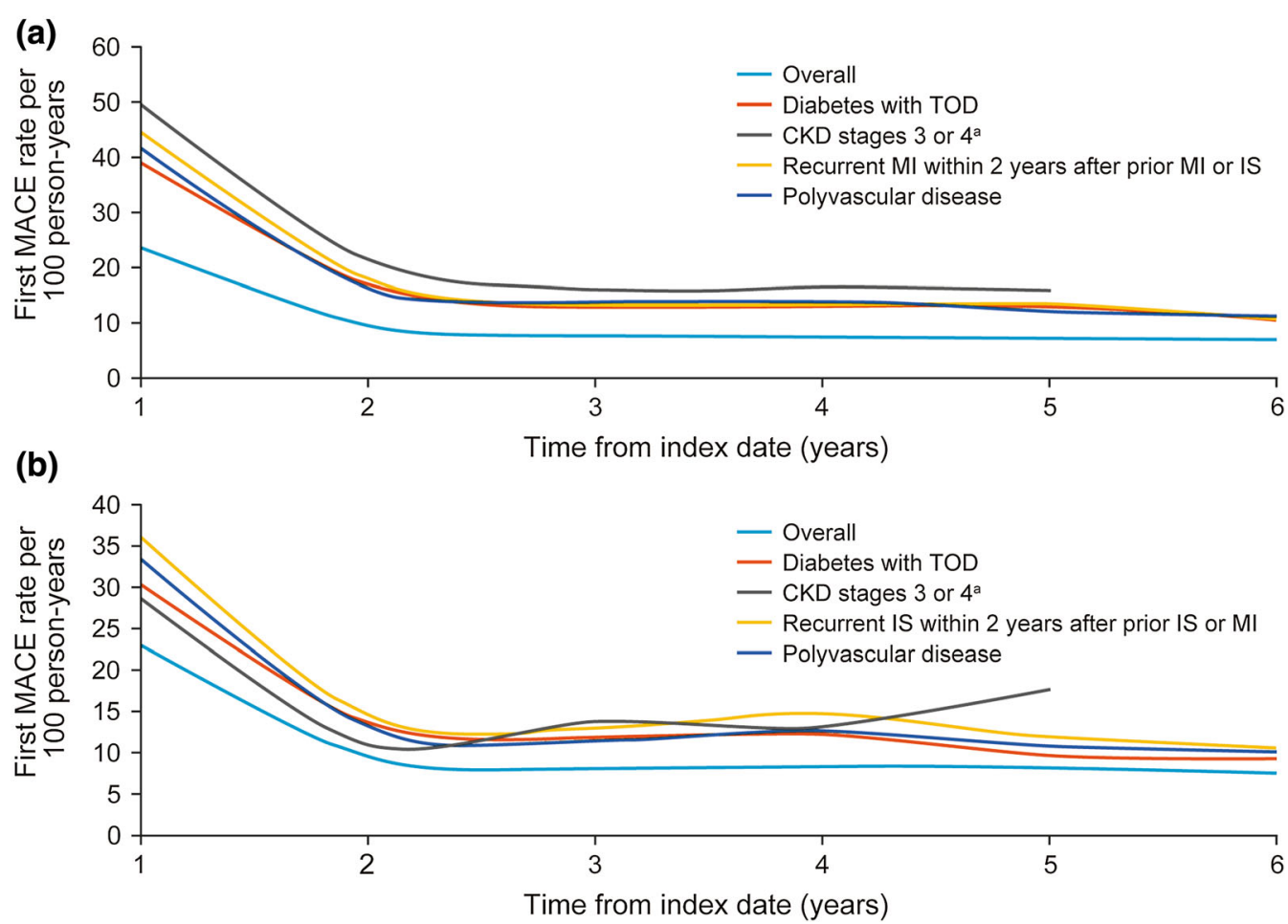

Fig. 3 First MACE yearly rates, overall and subgroups. a Patients with incident MI. b Patients with incident IS. ${ }^{a}$ Patient numbers in the CKD subgroup were too small for

(Amgen, data on file). The first MACE rate in patients with a recent IS in the placebo arm of FOURIER was 4.3 per 100 person-years, compared with 12.3 per 100 person-years in the incident IS cohort in the present study.

This very high risk of subsequent MACE in patients with MI or IS and additional risk factors indicates that there is a large unmet need with current disease management, and that additional interventions may be warranted, particularly early after an MI or IS. Indeed, this issue has been raised in European treatment guidelines, which recommend the use of aggressive secondary prevention interventions, including intense LDL-C-lowering treatment $[1,24]$. This is also consistent with a recent Swedish study that compared LDL-C levels achieved 6-10 weeks after an MI to the 2019 European Society for Cardiology/European Atherosclerosis Society guidelines LDL-C goal (below $1.4 \mathrm{mmol} / \mathrm{L}$ and at least a 50\% reduction in LDL-C [1]) and found that more than $80 \%$ of patients were eligible for analysis after year 5. CKD chronic kidney disease, IS ischaemic stroke, MACE major cardiovascular events, $M I$ myocardial infarction, TOD target organ damage

escalated lipid-lowering therapy [25]. Ensuring that patients with ASCVD and additional risk factors are offered intensified treatment known to decrease $\mathrm{CV}$ risk is crucial to avoiding a recurrence of $\mathrm{CV}$ events. In addition, a range of other preventive measures should be considered alongside lipid control, including encouraging adherence to lipid-lowering therapy, smoking cessation, diet and lifestyle advice, and, where relevant, control of hypertension and hyperglycaemia $[1,24]$.

Strengths of the present study include the long follow-up period, the large sample size, and the robustness of the data sets used, which cover all inpatient and specialised outpatient visits occurring in Sweden between 2006 and 2015. The National Patient Register contains more than $99 \%$ of hospitalisations, while the Prescribed Drug Register covers all prescriptions dispensed at pharmacies. This study, therefore, provides good estimates on event rates in patients in real-world clinical practice, rather 
than being based on highly selected clinical trial populations. As a result, our data could serve as a valuable source for populating health economic models evaluating interventions reducing $\mathrm{CV}$ risk.

Limitations of the study include the small sample size of some subgroups, and the lack of data on LDL-C levels, meaning that changes in $\mathrm{CV}$ risk could not be linked to the level of control of hyperlipidaemia. It is, however, well documented that the use of intensive lipid-lowering therapy is associated with CV risk reduction in very high-risk patients in clinical practice [26-28]. It should be noted that patients were included on the basis of the use of moderate- or high-intensity statins during the 1 year before the index date, but may have stopped or changed therapy by the index date. In addition, statin use, including adherence, and other concomitant therapies and interventions were not studied during follow-up, so potential treatment discontinuations and changes in type or intensity of treatment were not accounted for. It should also be noted that some very high-risk patients may reside in secondary care, such as rehabilitation facilities, and their statin use may not appear in a register of prescriptions. Furthermore, at the beginning of the study period, secondary prevention with high-intensity statins was not standard clinical practice in Sweden but became standard of care in 2012. This study was descriptive in nature. No statistical comparison of differences in rates between the subgroups was conducted, and further research to assess the statistical significance of the differences in rates might be of interest, in line with existing studies $[21,22]$. It might also be of interest to investigate the relevance of other risk factors that were not included in the present analysis, such as smoking or hypertension, because of the limitations of the data. Finally, it is important to note that, as this study was conducted in Sweden, the results might not be generalisable to other countries.

\section{CONCLUSION}

In conclusion, patients with very high CV risk with a history of MI or IS, and additional risk factors showed very high MACE rates despite previous use of lipid-lowering therapy with moderate- or high-intensity statins. Overall and in all subgroups, MACE rates were highest in the first 2 years following the index event and stable thereafter, highlighting the urgency of secondary prevention interventions early after an MI or IS to reduce the risk of subsequent MACE in these patient populations.

\section{ACKNOWLEDGEMENTS}

Funding. Open access funding provided by Uppsala University. This work, including payment of the journal's Rapid Service fee, was supported by Amgen.

Medical Writing, Editorial, and Other Assistance. Medical writing assistance was provided by Dan Booth PhD (Bioscript Medical Ltd, Macclesfield, UK) and funded by Amgen.

Author Contributions. All named authors meet the International Committee of Medical Journal Editors (ICMJE) criteria for authorship for this article, take responsibility for the integrity of the work as a whole, and have given their approval for this version to be published. ES and GV were responsible for data acquisition and analysis. All authors were involved in the study design, interpretation of results, and the drafting and critical review of the manuscript, and gave their approval for submission.

Disclosures. Emil Hagström acted as an expert committee member for and received lecture fees and institutional research grants from Sanofi and Amgen, and lecture fees from AstraZeneca, Bayer, and Novo Nordisk. Francesc Sorio Vilela and Guillermo Villa are employees and stockholders of Amgen. Maria K. Svensson was an employee and stockholder of Amgen when the study was performed. Sara Hallberg and Emma Söreskog are employees of Quantify Research, which was contracted and paid by Amgen to conduct the study.

Compliance with Ethics Guidelines. The study was performed in accordance with the 
Helsinki declaration of 1964 and its later amendments. Ethical approval for the present study was obtained from the Regional Ethical Review Board in Stockholm (dnr 2016/456-31/ 2 ). The need for individual patient consent was waived as a result of the study design.

Data Availability. All data generated or analysed during this study are included in this published article and as supplementary material online.

Open Access. This article is licensed under a Creative Commons Attribution-NonCommercial 4.0 International License, which permits any non-commercial use, sharing, adaptation, distribution and reproduction in any medium or format, as long as you give appropriate credit to the original author(s) and the source, provide a link to the Creative Commons licence, and indicate if changes were made. The images or other third party material in this article are included in the article's Creative Commons licence, unless indicated otherwise in a credit line to the material. If material is not included in the article's Creative Commons licence and your intended use is not permitted by statutory regulation or exceeds the permitted use, you will need to obtain permission directly from the copyright holder. To view a copy of this licence, visit http://creativecommons.org/licenses/by$\mathrm{nc} / 4.0 /$.

\section{REFERENCES}

1. Mach F, Baigent C, Catapano AL, et al. 2019 ESC/ EAS guidelines for the management of dyslipidaemias: lipid modification to reduce cardiovascular risk. Eur Heart J. 2020;41(1):111-88.

2. Mendis S. Global progress in prevention of cardiovascular disease. Cardiovasc Diagn Ther. 2017;7(Suppl 1):S32-8.

3. Townsend N, Wilson L, Bhatnagar P, Wickramasinghe K, Rayner M, Nichols M. Cardiovascular disease in Europe: epidemiological update 2016. Eur Heart J. 2016;37(42):3232-45.

4. American Heart Association: Cardiovascular disease: a costly burden for America. Projections through 2035. 2021. http://www.heart.org/idc/ groups/heart-public/@wcm/@adv/documents/down loadable/ucm_491543.pdf Accessed Feb 2021.

5. Roth GA, Fihn SD, Mokdad AH, Aekplakorn W, Hasegawa T, Lim SS. High total serum cholesterol, medication coverage and therapeutic control: an analysis of national health examination survey data from eight countries. Bull World Health Organ. 2011;89(2):92-101.

6. Bhatnagar D, Soran H, Durrington PN. Hypercholesterolaemia and its management. BMJ. 2008;337:a993.

7. Ference BA, Ginsberg HN, Graham I, et al. Lowdensity lipoproteins cause atherosclerotic cardiovascular disease. 1. Evidence from genetic, epidemiologic, and clinical studies. A consensus statement from the European Atherosclerosis Society Consensus Panel. Eur Heart J. 2017;38(32): 2459-72.

8. Baigent C, Blackwell L, Emberson J, et al. Efficacy and safety of more intensive lowering of LDL cholesterol: a meta-analysis of data from 170,000 participants in 26 randomised trials. Lancet. 2010;376(9753):1670-81.

9. Agabiti Rosei E, Salvetti M. Management of hypercholesterolemia, appropriateness of therapeutic approaches and new drugs in patients with high cardiovascular risk. High Blood Press Cardiovasc Prev. 2016;23(3):217-30.

10. Mahajan R. Real world data: additional source for making clinical decisions. Int J Appl Basic Med Res. 2015;5(2):82.

11. Rockberg J, Jørgensen L, Taylor B, Sobocki P, Johansson G. Risk of mortality and recurrent cardiovascular events in patients with acute coronary syndromes on high intensity statin treatment. Prev Med Rep. 2017;6:203-9.

12. Cherepanov D, Bentley TGK, Hsiao W, et al. Realworld cardiovascular disease burden in patients with atherosclerotic cardiovascular disease: a comprehensive systematic literature review. Curr Med Res Opin. 2018;34(3):459-73.

13. Gencer B, Mach F, Murphy SA, et al. Efficacy of evolocumab on cardiovascular outcomes in patients with recent myocardial infarction: a prespecified secondary analysis from the FOURIER trial. JAMA Cardiol. 2020;5(8):1-6.

14. Schwartz GG, Steg PG, Szarek M, et al. Alirocumab and cardiovascular outcomes after acute coronary syndrome. N Engl J Med. 2018;379(22):2097-107. 
15. Lindh M, Banefelt J, Fox KM, et al. Cardiovascular event rates in a high atherosclerotic cardiovascular disease risk population: estimates from Swedish population-based register data. Eur Heart J Qual Care Clin Outcomes. 2019;5(3):225-32.

16. Banefelt J, Lindh M, Svensson MK, Eliasson B, Tai $\mathrm{MH}$. Statin dose titration patterns and subsequent major cardiovascular events in very high-risk patients: estimates from Swedish population-based registry data. Eur Heart J Qual Care Clin Outcomes. 2020;6(4):323-31.

17. Ludvigsson JF, Almqvist C, Bonamy AK, et al. Registers of the Swedish total population and their use in medical research. Eur J Epidemiol. 2016;31(2):125-36.

18. Wallerstedt SM, Wettermark B, Hoffmann M. The first decade with the Swedish prescribed drug register-a systematic review of the output in the scientific literature. Basic Clin Pharmacol Toxicol. 2016;119(5):464-9.

19. Wettermark B, Hammar N, Fored CM, et al. The new Swedish Prescribed Drug Register-opportunities for pharmacoepidemiological research and experience from the first six months. Pharmacoepidemiol Drug Saf. 2007;16(7):726-35.

20. Ludvigsson JF, Andersson E, Ekbom A, et al. External review and validation of the Swedish national inpatient register. BMC Public Health. 2011;11:450.

21. Wilson PW, D'Agostino R Sr, Bhatt DL, et al. An international model to predict recurrent cardiovascular disease. Am J Med. 2012;125(7):695-703. e1.

22. Danese MD, Pemberton-Ross P, Catterick D, Villa G. Estimation of the increased risk associated with recurrent events or polyvascular atherosclerotic cardiovascular disease in the United Kingdom. Eur J Prev Cardiol. 2020;28:2047487319899212.
23. Sabatine MS, Giugliano RP, Keech AC, et al. Evolocumab and clinical outcomes in patients with cardiovascular disease. N Engl J Med. 2017;376(18): 1713-22.

24. Piepoli MF, Hoes AW, Agewall S, et al. 2016 European guidelines on cardiovascular disease prevention in clinical practice. The Sixth Joint Task Force of the European Society of Cardiology and Other Societies on Cardiovascular Disease Prevention in Clinical Practice (constituted by representatives of 10 societies and by invited experts. Developed with the special contribution of the European Association for Cardiovascular Prevention and Rehabilitation. G Ital Cardiol (Rome). 2017;18(7):547-612.

25. Allahyari A, Jernberg T, Hagström E, Leosdottir M, Lundman P, Ueda P. Application of the 2019 ESC/ EAS dyslipidaemia guidelines to nationwide data of patients with a recent myocardial infarction: a simulation study. Eur Heart J. 2020;41(40):3900-9.

26. Schiele F, Quignot N, Khachatryan A, et al. Clinical impact and room for improvement of intensity and adherence to lipid lowering therapy: five years of clinical follow-up from 164,565 post-myocardial infarction patients. Int J Cardiol. 2021;332:22-8.

27. Ahrens I, Khachatryan A, Monga B, Dornstauder E, Sidelnikov E. Association of treatment intensity and adherence to lipid-lowering therapy with major adverse cardiovascular events among post-MI patients in Germany. Adv Ther. 2021;38(5): 2532-41.

28. Khunti K, Danese MD, Kutikova L, et al. Association of a combined measure of adherence and treatment intensity with cardiovascular outcomes in patients with atherosclerosis or other cardiovascular risk factors treated with statins and/or ezetimibe. JAMA Netw Open. 2018;1(8):e185554.

29. Charlson ME, Pompei P, Ales KL, MacKenzie CR. A new method of classifying prognostic comorbidity in longitudinal studies: development and validation. J Chronic Dis. 1987;40(5):373-83. 